\title{
ELABORAÇÃO DE GRANOLA COM ADIÇÃO DE POLPA E CASTA- NHA DE BACURI PARA CONSUMO COM IOGURTE
}

\author{
Mendoza VS', Santos LL ${ }^{* *}$, Sanjinez-Argadoña EJ ${ }^{* * *}$
}

Resumo

A granola e o iogurte são produtos alimentícios que trazem benefícios para a saúde. A adição de frutos não convencionais com características nutricionais e sensoriais diferenciadas pode aumentar esses benefícios. O objetivo com este estudo foi avaliar a influência da adição do fruto do bacuri (Attalea phalerata Mart.) na formulação de granola para ser consumida com iogurte. As características microbiológicas, nutricionais e físicas da granola com bacuri e do iogurte foram determinadas. O produto foi avaliado sensorialmente utilizando o teste de diferença do controle e intenção de compra em 50 julgadores. A avaliação microbiológica demonstrou que o produto atende à Legislação vigente. $\mathrm{O}$ conteúdo de lipídeos foi significativamente maior que o da granola comercial, fornecendo um produto com alto valor energético. Os carotenoides da polpa de bacuri contribuíram com 10\% de Retinol na porção diária recomendada. A cor e a textura instrumental da granola também receberam influência do bacuri, resultando em um produto com tonalidade amarelo-laranja mais intensa e com maior cro-

* Graduanda em Engenharia de Alimentos pela Universidade Federal da Grande Dourados; vivianemendoza@hotmail.com

** Graduanda em Engenharia de Alimentos pela Universidade Federal da Grande Dourados; luanadeleon@hotmail.com

*** Doutora e Mestre em Engenharia de Alimentos pela Universidade Estadual de Campinas; Professora adjunta da Faculdade de Engenharia na Universidade Federal da Grande Dourados; Rodovia Dourados Itahum, Km 12, Unidade II, 79804-970, Dourados, Mato

Grosso do Sul, Brasil; elianajanet@gmail.com 
cância. Os testes sensoriais mostraram preferência pelo produto formulado com bacuri. Esses resultados permitem concluir que o fruto do bacuri é um ingrediente adequado para a elaboração de granola, uma vez que demonstrou melhoria nas propriedades de cor, textura e valor nutricional, e seu consumo com iogurte pode ser uma nova opção de alimento rápido e saudável.

Palavras-chave: Cereal matinal. Attalea phalerata Mart. Cerrado.

\title{
Granola preparation with addition of pulp and chestnut of bacuri for consumption with yogurt
}

\begin{abstract}
Granola and yogurt are food products beneficial to the health. The addition of non-conventional fruits with nutritional and differentiated sensorial characteristics may increase these benefits. In this way, this paper pursues the utilization of bacuri fruit (Attalea phalerata Mart.) in the production of granola to be consumed with yogurt. The physics, microbial, and nutritional characteristics of granola with bacuri and yogurt were determinate. The product was sensorial evaluated by the difference from a control test and intention purchase. The tests were applied to 50 judges. The microbiological evaluation showed that the product complies with current Legislation. Lipid content was significantly higher than that of commercial granola, providing a high energy product. Carotenoids from bacuri pulp contributed with 10\% of Retinol in the recommended daily dose. The color and instrumental texture of the granola were also given the same influence, resulting in a product with a more intense yellow-orange hue and with greater crispness. Sensory tests show preference for product formulated with bacuri. These results allow to conclude that bacuri fruit is an ingredient adequate to granola manufacturing, once it demonstrated the improvement in the proprieties such as, color, texture, nutritional and energy value of product comparing with commercial granola. Therefore, the bacuri granola to consume with yogurt may be a new option of fast and healthy food.
\end{abstract}

Keywords: Matinal cereal. Attalea phalerata Mart. Cerrado.

\section{INTRODUÇÃO}

Os produtos alimentícios que apresentam influência na saúde humana estão em destaque no mercado diante da crescente preocupação dos consumidores em relação ao estilo de vida e saúde. A divulgação na mídia de descobertas científicas que evidenciam a incidência de morte em razão de acidentes cardiovasculares, câncer, acidente vascular cerebral, arteriosclerose, enfermidades hepáticas, entre outros, causados na maioria das vezes por maus hábitos, ${ }^{1}$ contribui para a escolha do alimento. Assim, o interesse por alimentos mais saudáveis ou com alegações funcionais está relacionado às atuais necessidades em prevenir problemas de saúde.

Entre os produtos que promovem benefícios para a saúde destacam-se a granola e o iogurte. A granola fornece principalmente fibras solúveis e insolúveis. As fibras solúveis contribuem para a diminuição do nível de colesterol e atuam no combate à obesidade. Enquanto as fibras insolúveis aceleram a velocidade do trânsito fecal e estimulam o bom funcionamento intestinal. ${ }^{2}$ 
A granola é um produto alimentar à base de cereais e ingredientes como aveia, nozes e mel, que são consumidos, geralmente, como cereal matinal. ${ }^{3}$ Constituída principalmente por carboidratos, é um produto energético que pode ser consumido como suplemento, fornecendo parte da energia que o corpo necessita para executar as atividades diárias e manter o funcionamento adequado de todo o organismo. Os cereais integrais, grãos e sementes na elaboração da granola podem fornecer um produto com menor índice glicêmico em razão dos carboidratos complexos, que são mais lentamente digeridos, liberando menos açúcar no sangue em comparação a outras fontes de carboidratos simples, como o açúcar refinado, presente frequentemente em bebidas prontas e produtos ultraprocessados. ${ }^{4}$

No Cerrado sul-mato-grossense estão inseridas diversas espécies frutíferas, que produzem frutos comestíveis de cores atrativas e sabores característicos. Entre essas espécies, a Attalea phalerata Mart., conhecida popularmente como acuri, bacuri e urucuri, ${ }^{5}$ destaca-se quanto ao potencial agrícola, pois pode se desenvolver em solos pobres em nutrientes e com déficit hídrico. Seus frutos são constituídos de polpa e sementes comestíveis com elevado valor nutricional. ${ }^{6}$

Segundo autores pesquisados, ${ }^{7}$ o bacuri está entre os frutos que se destacam em relação à atividade antioxidante dos compostos fenólicos e dos carotenoides, presentes principalmente na polpa. $\mathrm{O}$ efeito benéfico desses compostos bioativos à saúde desperta o interesse da indústria e da comunidade científica. Entre as atividades biológicas atribuídas a esses compostos estão o fortalecimento do sistema imunológico ${ }^{8}$ e a diminuição de doenças degenerativas, como o diabetes, problemas cardiovasculares e processos inflamatórios. ${ }^{9}$ Diante disso, a utilização racional de espécies frutíferas do Cerrado, como os frutos da Attalea phalerata, na elaboração de produtos alimentícios com alegações funcionais pode contribuir para o desenvolvimento econômico e sustentável da região. Além disso, contribui para o incentivo à promoção da saúde por meio da inclusão de alimentos regionais mais saudáveis na dieta. ${ }^{10,11}$

O consumo de alimentos regionais é recomendado pela Organização Mundial de Saúde e pelo Ministério da Saúde no Guia Alimentar para a População Brasileira ${ }^{12}$ como umas das estratégias para a promoção da alimentação saudável e a redução do risco de doenças crônicas não transmissíveis (DCNT). A inserção desses alimentos na alimentação habitual também contribui para a aplicação do conceito ampliado de segurança alimentar e nutricional, que envolve o abastecimento e acesso universal aos alimentos, somado aos seus aspectos nutricionais e de qualidade e ao manejo sustentável dos recursos naturais. ${ }^{13}$ Nessa perspectiva, o objetivo neste trabalho foi elaborar granola com adição de polpa e castanha de bacuri e disponibilizá-la para o consumidor juntamente com iogurte.

\section{MATERIAL E MÉTODOS}

\subsection{MATERIAL}

Frutos de bacuri (Attalea phalerata Mart.) coletados no Município de Bonito, MS (Hotel Cabanas), óleo de girassol, aveia em flocos, mel, linhaça e flocos de milho sem açúcar foram empregados na elaboração da granola. Para efeito de comparação, foi adquirida granola comercial composta pelos 
seguintes ingredientes: aveia em flocos, flocos de trigo, mel, flocos de arroz, linhaça dourada, castanha do Pará e óleo de girassol.

Cultura láctea, açúcar, leite integral e em pó foram utilizados na elaboração do iogurte. Todos os ingredientes, com exceção dos frutos de bacuri, foram adquiridos no mercado local.

\subsection{MÉTODOS}

A elaboração dos produtos (granola e iogurte) e as análises microbiológicas, físicas, químicas e sensoriais foram realizadas nos Laboratórios do curso de Engenharia de Alimentos da Universidade Federal da Grande Dourados (UFGD).

\subsubsection{Obtenção da polpa e da castanha de bacuri}

Frutos sadios foram lavados em água corrente e higienizados com solução de dicloroisociarunato de sódio diihidratado $0,66 \%$ (teor de cloro ativo $3 \%$ ), descascados e despolpados manualmente. A polpa em pedaços foi desidratada em estufa com circulação de ar a $40{ }^{\circ} \mathrm{C}$ por 72 horas. As castanhas, extraídas em prensa hidráulica (MARCON-MPH15), foram desidratadas a $70^{\circ} \mathrm{C}$ por três horas.

\subsubsection{Processamento}

A granola foi elaborada pela mistura de ingredientes sólidos e líquidos. As etapas de processamento são mostradas na Figura 1. A elaboração do iogurte consistiu na fermentação láctica do leite de vaca mediante a ação de Lactobacillus bulgaricus e Streptococcus thermophilus; as etapas envolvidas na elaboração estão presentes na Figura 2.

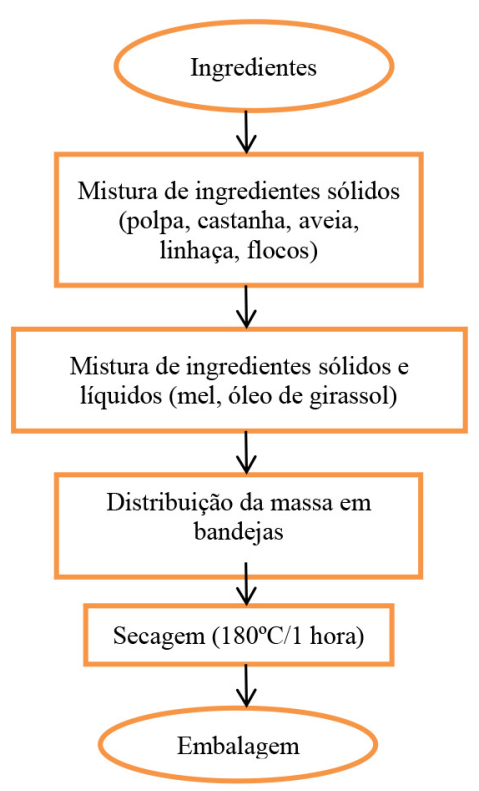

Figura 1 - Etapas do processamento para obtenção da granola 


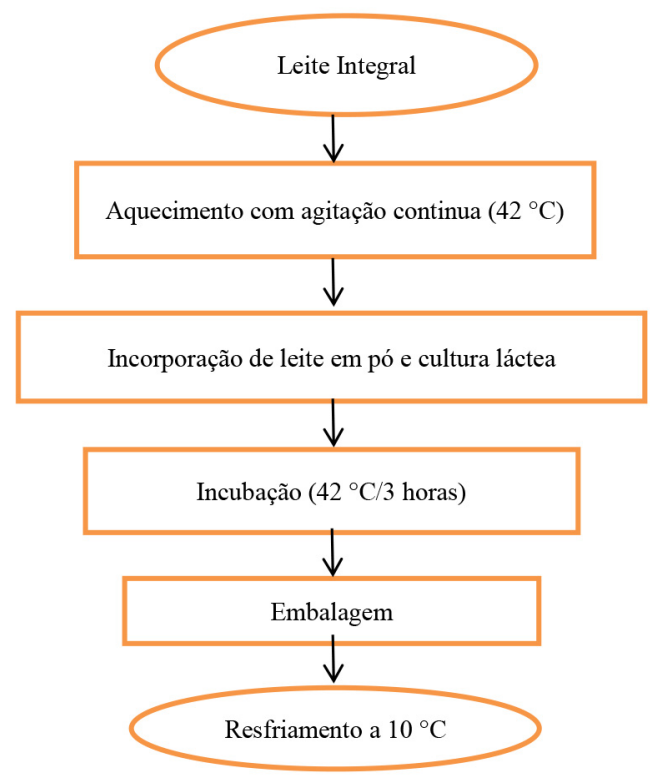

Figura 2 - Etapas do processamento para obtenção do iogurte

\subsubsection{Análises microbiológicas}

Coliformes totais e termotolerantes foram analisados na granola e no iogurte. Determinações de Bacillus cereus, Staphylococcus aureus e Salmonella spp. foram realizadas na granola de acordo com os procedimentos descritos por Silva. ${ }^{14}$ As análises foram realizadas com base nos Padrões Microbiológicos para Alimentos da Legislação vigente, estabelecida pela Resolução RDC n. $12 .{ }^{15}$

\subsubsection{Análises físicas e químicas}

As determinações de umidade, lipídeos, proteína, fibras, minerais, $\mathrm{pH}$ e acidez foram realizadas de acordo com os procedimentos descritos nas Normas Analíticas do Instituto Adolfo Lutz. ${ }^{16} \mathrm{O}$ teor de carboidratos foi estimado por diferença, diminuindo de 100 o somatório de umidade, lipídeos, proteínas, fibras e minerais. A atividade de água foi determinada com o auxílio do higrômetro Aqualab à temperatura de $25^{\circ} \mathrm{C}$, previamente calibrado.

Os carotenoides totais, presentes na granola, foram quantificados de acordo com o procedimento descrito por Rodriguez-Amaya ${ }^{9}$ calculados pela Equação 1.

$$
C \mathrm{E} \mu g / g \mathrm{~F} \mathrm{Z} \frac{\lambda^{\sim} D^{\sim} \mathrm{MT}}{\varepsilon^{\sim} m \mathrm{E} g \mathrm{~F}}
$$

Em que C é a quantidade de carotenoides ( $\mu \mathrm{g} / \mathrm{g}), \lambda$ é a absorbância (nm), D é a diluição ( $\mathrm{ml}$ ), $\varepsilon$ é o fator de $\beta$-caroteno em éter de petróleo (2592) e m é a massa da amostra (g). 


\subsubsection{Valor energético}

O valor energético foi calculado a partir dos dados da composição nutricional, de acordo com a RDC n. 360 do Ministério da Saúde. ${ }^{17}$ No cálculo foram considerados os fatores de conversão de $4 \mathrm{kcal} / \mathrm{g}$ para carboidratos e proteínas e de $9 \mathrm{kcal} / \mathrm{g}$ para lipídios, e expressos em kcal/g e kJ/g.

\subsubsection{Análise de cor instrumental}

Foram determinados os parâmetros de cor $\mathrm{L}^{\star}$ que define a claridade $\left(\mathrm{L}^{*}=0\right.$ preto e $\mathrm{L}^{\star}=100$ branco), $a^{\star}$ (- $a^{\star}$ verde $e+a^{\star}$ vermelho) e $b^{\star}\left(-b^{\star}\right.$ azul $e+b^{\star}$ amarelo). A partir desses resultados foram calculados os parâmetros de saturação $\left(C^{\star}\right)$, que definem a intensidade da cor da amostra (Equação 2), a tonalidade $\left(\mathrm{h}^{\circ}\right)$ (calculada pela Equação 3) e a variação de cor $(\Delta \mathrm{E})$, que expressa a diferença da cor entre as amostras (Equação 4). As determinações foram feitas por leitura direta, com seis repetições, utilizando colorímetro Konica Minolta (modelo CR410) a $25^{\circ} \mathrm{C}$ no sistema de cores CIELAB.

$$
\begin{gathered}
\mathrm{C}^{*}=\sqrt{\mathrm{a}^{* 2}+\mathrm{b}^{* 2}} \\
\mathrm{~h}^{\circ}=\arctan \frac{\mathrm{b}^{*}}{\mathrm{a}^{*}} \\
\Delta \mathrm{E}=\sqrt{\left(\mathrm{L}_{0}^{*}-\mathrm{L}^{*}\right)^{2}-\left(\mathrm{a}_{0}^{*}-\mathrm{a}^{*}\right)^{2}-\left(\mathrm{b}_{0}^{*}-\mathrm{b}^{*}\right)^{2}}
\end{gathered}
$$

Em que $\mathrm{a}^{\star}, \mathrm{b}^{\star}$ e $\mathrm{L}^{\star}$ são os parâmetros de cromaticidade e claridade da granola com bacuri, e $\mathrm{a}_{0}^{*}, \mathrm{~b}_{0}^{*} \mathrm{e} \mathrm{L}_{0}^{*}$ são os parâmetros da granola comercial.

\subsubsection{Análise de textura instrumental}

A crocância da granola foi analisada pelo teste de compressão avaliando-se a força de compressão em analisador de textura TAHDI (Stable MicroSystems) calibrado com célula de carga de $5 \mathrm{~kg}$. A amostra (30 gramas) foi colocada em um pot base inserts, e a compressão foi realizada utilizando-se placa cilíndrica com diâmetro de $5 \mathrm{~cm}$. A distância da compressão foi de $10 \mathrm{~mm}$ em relação à amostra, com velocidade constante de 5,0 mm/s. Foram realizadas nove repetições.

\subsubsection{Avaliação sensorial}

Foi realizado o teste de diferença do controle com 50 julgadores não treinados, que avaliaram o grau de diferença entre as amostras de iogurte servidas com granola com bacuri e com granola comercial (amostra de referência). Foram avaliados cor, sabor, textura, aroma e aceitação global das amostras por meio da escala de notas no intervalo de -4 (gostei muitíssimo menos que a R) e 4 (gostei muitíssimo mais que a R) empregando uma ficha sensorial (Figura 3). Avaliou-se também a intenção de compra do iogurte com granola com bacuri. 
Nome:
Sexo: ( )Feminino ( )Masculino $\quad$ Idade: ( ) $<24$ ( )25-35 ( )36-50 ( )>50

Você está recebendo duas amostras de granola com iogurte, avalie inicialmente a amostra com a letra $\mathrm{R}$ e depois a amostra codificada e expresse o quanto você gostou ou desgostou da amostra codificada em relação aos atributos cor, aroma, textura, sabor e aceitação global, utilizando a escala de notas abaixo.

\begin{tabular}{|c|c|c|c|c|c|c|}
\hline $\begin{array}{l}\text { Notas } \\
\text {-4) Gostei muitíssimo menos que a } R \\
\text {-3) Gostei muito menos que a } R \\
\text {-2) Gostei regularmente menos que a } R \\
\text {-1) Gostei ligeiramente menos que a } R \\
\text { 0) Gostei da amostra igualmente a } R \\
\text { 1) Gostei ligeiramente mais que a } R \\
\text { 2) Gostei regularmente mais que a } R \\
\text { 3) Gostei muito mais que a } R \\
\text { 4) Gostei muitíssimo mais que a } R\end{array}$ & $\begin{array}{l}\text { N. de } \\
\text { Amostra }\end{array}$ & Cor & Aroma & Textura & Sabor & Aceitação global \\
\hline
\end{tabular}
Figura 3 - Modelo da ficha sensorial do teste diferença do controle

\subsubsection{Análise estatística}

Os resultados foram expressos pelo valor médio das repetições e o desvio padrão. Eles foram submetidos à análise da variância (ANOVA), com comparação de médias pelo teste de Tukey ao nível de 5\% de significância, com o auxílio do programa STATISTICA 8.0.

\section{RESULTADOS E DISCUSSÃO}

A formulação da granola com adição de polpa e castanha de bacuri encontra-se na Tabela 1 . A granola foi constituída por 52\% de ingredientes secos (polpa de bacuri, aveia, castanha de bacuri, flocos de milho e linhaça) e 48\% de ingredientes líquidos (mel de abelha e óleo de girassol). Da massa total do produto, o mel de abelha representa $28 \%$. As propriedades funcionais do mel, como a atividade prebiótica, que tem efeito no trânsito intestinal e na regulação da pressão arterial e dos níveis de colesterol, justificam sua adição. ${ }^{18}$

Tabela 1 - Formulação de granola com adição de polpa e castanha de bacuri

\begin{tabular}{cc}
\hline Ingredientes & Quantidade (\%) \\
\hline Polpa de bacuri & 28 \\
Aveia & 9 \\
Castanha de bacuri & 9 \\
Flocos de milho & 3 \\
Linhaça & 3 \\
Mel de abelha & 28 \\
Óleo de girassol & 20 \\
\hline
\end{tabular}


A granola e o iogurte foram formulados separadamente, mas no momento da ingestão devem ser consumidos misturados. O iogurte contribui principalmente com o aroma, sabor e viscosidade, ${ }^{19}$ já a granola é rica em carboidratos, fibras e minerais, além de apresentar atributos sensoriais de cor, aroma, sabor e crocância. ${ }^{20}$ Todos esses atributos em um só produto podem atender aos consumidores que buscam alimentos práticos para o dia a dia, conciliando com o bem-estar e a saúde.

\subsection{AVALIAÇÃO MICROBIOLÓGICA}

Os coliformes totais e termotolerantes presentes na granola com bacuri foram inferiores a 3 NMP. $g^{-1}$ (Tabela 2). Para coliformes, a Legislação estabelece parâmetro somente para os termotolerantes, e o resultado obtido está de acordo com ela. Isso mostra que as condições higiênico-sanitárias do processamento foram eficazes. Para Bacillus cereus e Staphylococcus aureus (Tabela 2), os valores atendem à Legislação brasileira. ${ }^{15}$ A microbiota, geralmente presente em granolas, é constituída por micro-organismos do solo e do ambiente em que estão armazenados. Quando as condições permitem o desenvolvimento microbiano, as bactérias do gênero Bacillus spp. são os principais micro-organismos que se desenvolvem em cereais, por serem produtores de amilase. ${ }^{21}$

A ausência de matéria-prima de origem animal reduz a probabilidade de contaminação por Salmonella spp., porém granolas que contêm ingredientes como amendoim, coco, nozes e passas de frutas podem apresentar contaminação por esse micro-organismo advindo desses ingredientes. ${ }^{22}$ Esses resultados indicam que a granola elaborada com polpa e castanha de bacuri apresenta qualidade microbiológica adequada para o consumo e comercialização.

Tabela 2 - Características microbiológicas da granola com bacuri e do iogurte elaborados e os padrões estabelecidos pela legislação brasileira

\begin{tabular}{lcccc}
\hline \multirow{2}{*}{\multicolumn{1}{c}{ Análises }} & \multicolumn{2}{c}{ Produto elaborado } & \multicolumn{2}{c}{ Padrões brasileiros } \\
\cline { 2 - 5 } & Granola1 $^{\dagger}$ & Iogurte & Granola & Iogurte \\
\hline Coliformes totais (NMP.g-1) & $<3,0$ & 7,2 & - & - \\
Coliformes termotolerantes (NMP.g-1) & $<3,0$ & $<3,0$ & $<5,0 \times 10^{2}$ & 10,0 \\
Bacillus cereus (UFC.g-1) & $<1,0 \times 10^{2}$ & - & $<5,0 \times 10^{3}$ & - \\
Staphylococcus aureus (UFC. g-1) & $<1,0 \times 10^{1}$ & - & $<1,0 \times 10^{3}$ & - \\
Salmonella spp. em 25g & Ausência & - & Ausência & - \\
\hline
\end{tabular}
Fonte: Brasil. ${ }^{15}$

No iogurte, a Legislação somente indica valores microbiológicos para coliformes termotolerantes ${ }^{15}$ que são encontrados normalmente no intestino de homens e animais. Por conta disso, a contaminação de um alimento por esse grupo de microrganismos indica manipulação inadequada durante o processamento da matéria-prima ou higienização inadequada de equipamentos. ${ }^{23} \mathrm{O}$ iogurte desenvolvido neste trabalho atendeu à Legislação, pois o valor obtido foi <3,0 NMP.g-1 .

\footnotetext{
${ }^{\dagger}$ Granola elaborada com adição de polpa e castanha de bacuri.
} 


\subsection{CARACTERÍSTICAS FÍSICAS E QUÍMICAS}

O teor de umidade da granola com bacuri foi menor que o obtido por outros autores ${ }^{22,24}$ para granola comercial (Tabela 3). O baixo conteúdo de umidade se deve ao processo de secagem, no qual ocorre a eliminação da água através da evaporação, ${ }^{25}$ o que favorece a vida útil do produto, desde que embalado adequadamente. Esta afirmativa pode ser ratificada pela baixa atividade de água $(0,477)$.

Tabela 3 - Composição nutricional, acidez, pH e atividade de água da granola com adição de polpa e castanha de bacuri

\begin{tabular}{lrrr}
\hline \multicolumn{1}{c}{ Constituintes } & Granola2 $^{\ddagger}$ & Autores 122 & Autores 224 \\
\hline Valor energético $(\mathrm{kcal})$ & 449,06 & 378,49 & - \\
Valor energético $(\mathrm{kJ})$ & 1884,03 & - & - \\
Umidade $(\mathrm{g} / 100 \mathrm{~g})$ & $3,61 \pm 0,38$ & 9,17 & 5,94 \\
Lipídeos $(\mathrm{g} / 100 \mathrm{~g})$ & $19,58 \pm 1,15$ & 5,73 & 15,9 \\
Proteína $(\mathrm{g} / 100 \mathrm{~g})$ & $7,10 \pm 0,86$ & 9,4 & 12,4 \\
Fibra insolúvel $(\mathrm{g} / 100 \mathrm{~g})$ & $6,58 \pm 0,17$ & 1,58 & - \\
Minerais $(\mathrm{g} / 100 \mathrm{~g})$ & $2,00 \pm 0,05$ & 1,79 & 63,82 \\
Carboidratos $(\mathrm{g} / 100 \mathrm{~g})$ & $61,11 \pm 0,00$ & 72,33 & - \\
Acidez titulável $(\mathrm{g} / 100 \mathrm{~g})$ & $0,11 \pm 0,01$ & 0,32 & - \\
pH & $5,52 \pm 0,02$ & 4,98 & - \\
Atividade de água & $0,477 \pm 0,01$ & - & - \\
Carotenoides totais $(\mu \mathrm{g} / 15 \mathrm{~g})$ & 360,45 & - &
\end{tabular}

Alimentos com atividade de água (Aw) até 0,60 são classificados como alimentos com baixa umidade. Nesse intervalo de Aw o crescimento de micro-organismos é mínimo, porém podem ocorrer reações químicas de oxidação, hidrólise, entre outras ${ }^{26}$, que alteram o produto.

Segundo autores pesquisados, ${ }^{27,28}$ a atividade de água e a umidade relativa do ambiente tendem a equilibrar-se, portanto alimentos com Aw inferior à umidade relativa do ambiente em que estão armazenados tendem a absorver água, o que poderá acarretar perda de atributos sensoriais importantes como crocância, sendo necessário o acondicionamento em embalagem adequada.

O alto teor de lipídeos da granola com bacuri pode ser justificada pela adição de óleo de girassol e da castanha de bacuri. A castanha de bacuri apresenta alto conteúdo de ácido graxo láurico seguido do ácido graxo monoinsaturado oleico. ${ }^{29} \mathrm{O}$ ácido láurico é altamente oxidado no corpo, ${ }^{30}$ assim, inserido na classe dos ácidos graxos de cadeia média (AGCM) aumenta o gasto energético, oxida a gordura e reduz depósitos de gordura e a massa corporal. ${ }^{31} \mathrm{O}$ ácido oleico é um importante ácido graxo monoinsaturado de rápida absorção e redutor da massa corporal. ${ }^{32}$

A discrepância do teor de proteína entre a granola formulada e o apresentado por Souza et al. ${ }^{24}$ (Tabela 4) pode estar relacionada aos diferentes ingredientes utilizados na elaboração de cada for-

\footnotetext{
${ }^{\ddagger}$ Granola elaborada com adição de polpa e castanha de bacuri.
} 
mulação de granola. Porém, os resultados obtidos apresentaram pouca diferença em relação ao obtido pelos autores $1 .^{22}$

O teor de fibras da granola com bacuri foi maior que os apresentados pelos autores $1 .{ }^{22}$ As fibras têm importante função na saúde, pois aceleram a passagem dos produtos residuais do organismo, absorvem toxinas e mantêm o tubo digestivo saudável. Além disso, auxiliam na formação de ácidos graxos de cadeia curta, fornecendo energia para as células intestinais desempenharem bem suas funções. ${ }^{33}$ A recomendação diária de consumo de fibras é de $25 \mathrm{~g}$, considerando uma dieta de $2000 \mathrm{kcal} .{ }^{34}$ A granola com bacuri na porção recomendada de 15 g contém $1 \mathrm{~g}$ de fibras, o que corresponde a 4\% da recomendação diária.

No que se refere ao teor de carboidratos, a granola com adição de bacuri não apresentou diferença em relação à granola comercial. O alto teor de carboidratos $(61,11 \mathrm{~g} / 100 \mathrm{~g})$ define o produto como fonte energética. ${ }^{4}$

Os valores obtidos para os minerais foram semelhantes aos encontrados pelos autores pesquisados. ${ }^{22,24}$ A granola com bacuri pode ser considerada pouco ácida em razão do $\mathrm{pH}(5,5)$ e do baixo teor de acidez $(0,11 \mathrm{~g} / 100 \mathrm{~g})$.

Estudos fitoquímicos mostram que a polpa desidratada do bacuri apresenta 24,03 $\mu \mathrm{g}$ de $\beta$-caroteno em $1 \mathrm{~g}$ de amostra. ${ }^{6}$ Neste trabalho, a quantidade de granola sugerida para o consumo (15 g) apresentou 360,45 $\mu \mathrm{g}$ de $\beta$-caroteno, essa quantidade equivale a 60,20 $\mu \mathrm{g}$ de retinol (RE).

Os carotenoides são precursores alimentares do retinol, com atividade biológica de pró-vitamina A. ${ }^{35}$ A vitamina A é importante para o crescimento, desenvolvimento, manutenção de tecidos epiteliais, reprodução, sistema imunológico e, em especial, para o funcionamento do ciclo visual na regeneração de fotorreceptores. ${ }^{36}$ Vários estudos têm demonstrado ação anticarcinogênica, imunomoduladora e preventiva de doenças cardiovasculares. ${ }^{37,38}$

De acordo com a Anvisa, ${ }^{39}$ a ingestão diária de retinol recomendada para adultos é de $600 \mu \mathrm{g}$. Embora o consumo de $15 \mathrm{~g}$ de granola represente 10\% da IDR, a contribuição do produto pode ser relevante em uma dieta balanceada. Além disso, percebe-se a contribuição dos carotenoides da polpa do bacuri na cor do produto, tornando-o mais atrativo.

Os constituintes nutricionais do iogurte são apresentados na Tabela 4. Os valores de umidade, lipídios, proteína, fibras, minerais e $\mathrm{pH}$ foram semelhantes aos da Tabela Brasileira de Composição de Alimentos (TACO), ${ }^{40}$ e os valores de lipídeos, proteína e acidez também estão de acordo com os definidos pela legislação brasileira. ${ }^{41}$

A alta atividade de água do iogurte $(0,960)$ indica condições favoráveis de multiplicação para a maioria de bactérias e fungos deteriorantes. Porém, o pH abaixo de 4,5 é uma barreira para o crescimento microbiano. ${ }^{42}$ 
Tabela 4 - Composição nutricional, acidez, pH e atividade de água do iogurte elaborado e do referencial teórico obtidos da Tabela Brasileira de Composição de Alimentos e do Ministério de Agricultura e do Abastecimento

\begin{tabular}{lrrr}
\hline \multicolumn{1}{c}{ Constituintes } & Iogurte elaborado & TACO (2011) & MAPA (2007) \\
\hline Valor energético $(\mathrm{kcal})$ & 53,39 & 51 & - \\
Valor energético $(\mathrm{kJ})$ & 222,67 & 215 & - \\
Umidade $(\mathrm{g} / 100 \mathrm{~g})$ & $89,85 \pm 0,31$ & 90 & - \\
Lipídeos $(\mathrm{g} / 100 \mathrm{~g})$ & $3,39 \pm 0,08$ & 4,1 & $3,0-5,9$ \\
Proteína $(\mathrm{g} / 100 \mathrm{~g})$ & $4,45 \pm 0,22$ & 0,9 & Mín. 2,9 \\
Minerais $(\mathrm{g} / 100 \mathrm{~g})$ & $1,04 \pm 0,04$ & 1,9 & - \\
Carboidratos $(\mathrm{g} / 100 \mathrm{~g})$ & $1,27 \pm 0,00$ & - & - \\
\hline Acidez titulável $(\mathrm{g} / 100 \mathrm{~g})$ & $0,86 \pm 0,00$ & - & - \\
pH & $4,00 \pm 0,04$ & - & - \\
Atividade de água & $0,96 \pm 0,00$ & & - \\
\hline Fonte: adaptado de Tabela Brasileira de Composição de Alimentos ${ }^{40}$ e Ministério da Agricultura, Pecuária e Abastecimento. & ${ }^{41}$
\end{tabular}

Entre os principais benefícios do iogurte destacam-se o fortalecimento do sistema imunológico e da saúde óssea e a prevenção de doenças gastrointestinais, osteoporose, alergias e intolerâncias ao leite, além de ser uma forma indireta do consumo de leite. $\mathrm{O}$ consumo do iogurte está ligado à imagem de alimento saudável e nutritivo, associado às suas qualidades sensoriais. ${ }^{43,44}$

A granola com adição de bacuri e iogurte, na porção recomendada de $15 \mathrm{~g}$ de granola e $110 \mathrm{~g}$ de iogurte, contêm 67,36 kcal e 58,73 kcal, respectivamente. Segundo a Associação Portuguesa dos Nutricionistas, ${ }^{45}$ essa quantidade de valor energético para o iogurte o classifica como magro, e não foram encontradas informações brasileiras referentes a essa classificação. Juntos (granola e iogurte) apresentam $126,09 \mathrm{kcal}(527,54 \mathrm{~kJ})$, o que representa 6,3\% do valor energético diário recomendado para uma pessoa adulta (2000 kcal ou $8400 \mathrm{~kJ}),{ }^{46}$ sendo considerado um produto com baixo valor energético.

\subsection{AVALIAÇÃO DA COR}

A adição de bacuri na granola influenciou a cor do produto, que se apresentou amarelo-laranja significativamente $(\mathrm{P}<0,05)$ mais intensa que a granola comercial, observada pelos maiores valores de $\mathrm{a}^{\star}$ e de $\mathrm{b}^{\star}$ (Tabela 5). Esses resultados eram esperados, uma vez que o amarelo-laranja é a cor característica da polpa de bacuri, portanto, a granola comercial ficou mais clara (maior valor de $\mathrm{L}^{\star}$ ).

A intensidade da cor amarela também foi verificada pela saturação da cor (maior valor de $\left.\mathrm{C}^{\star}\right)$. No que se refere à tonalidade $\left(\mathrm{h}^{\circ}\right)$, valores menores indicam que o produto apresenta tonalidade próxima à cor vermelha, proporcionando, neste caso, cor alaranjada. A tonalidade da cor foi influenciada pela polpa de bacuri, o mel e a caramelização dos açúcares. 
Tabela 5 - Parâmetros de cor instrumental de cromaticidade $\left(\mathrm{a}^{\star}, \mathrm{b}^{*}\right)$, claridade $\left(\mathrm{L}^{\star}\right)$, saturação $\left(\mathrm{C}^{*}\right)$ e tonalidade $\left(\mathrm{h}^{\star}\right)$ da granola comercial e da granola com bacuri ${ }^{*}{ }^{\star s}$

\begin{tabular}{crr}
\hline Parâmetros & Granola comercial & Granola com bacuri \\
\hline $\mathrm{L}^{*}$ & $51,52^{\mathrm{a}}$ & $34,66^{\mathrm{b}}$ \\
$\mathrm{a}^{*}$ & $6,55^{\mathrm{a}}$ & $11,33^{\mathrm{b}}$ \\
$\mathrm{b}^{*}$ & $22,56^{\mathrm{a}}$ & $28,74^{\mathrm{b}}$ \\
$\mathrm{C}^{*}$ & $23,51^{\mathrm{a}}$ & $31,17^{\mathrm{b}}$ \\
$\mathrm{h}^{*}$ & $1,29^{\mathrm{a}}$ & $1,18^{\mathrm{b}}$ \\
\hline
\end{tabular}

As amostras de granola diferiram-se entre si ao nível de 5\% de significância pelo teste de Tukey em todos os parâmetros de cor. O valor total da diferença da cor $(\Delta \mathrm{E})$ representa a tolerância que pode ser permitida sem afetar a percepção das diferenças.

Segundo Moritz ${ }^{47}$ as tolerâncias industriais, de acordo com a escala de cor CIELAB, mantêm-se em uma faixa de 0,2 até 2,5 . Na indústria esse parâmetro é muito útil no controle de qualidade de produtos ou no desenvolvimento de novos produtos. $\mathrm{O} \Delta \mathrm{E}$ da granola com bacuri foi de 16,86 em relação à granola comercial. Portanto, pode-se afirmar que a granola com bacuri apresentou cor diferente da granola comercial.

\subsection{AVALIAÇÃO DA TEXTURA}

Os valores médios da força de compressão apresentaram diferenças significativas $(\mathrm{P}<0,05)$, contudo, na Tabela 6, observam-se dois conjuntos de valores (1279,5 g a $1979 \mathrm{~g}$ e $3024,4 \mathrm{~g}$ a $3865,2 \mathrm{~g}$ ).

Algumas amostras da granola apresentaram maior resistência à compressão, provavelmente por conterem maior quantidade de castanha e polpa de bacuri, esses ingredientes apresentam maior resistência à compressão, ou seja, são mais rígidos que os flocos de milho ou de aveia. Por outro lado, o conjunto de valores menores pode ser justificado pela maciez e irregularidade dos flocos de milho, compactação e espaços vazios (pot base) que a sonda do analisador de textura detecta ao realizar a compressão da amostra.

Correlacionando-se a força necessária para quebrar a amostra durante a compressão com a crocância, pode-se inferir que a granola com bacuri apresentou uma textura crocante aceitável para a mastigação humana. Na análise sensorial os julgadores consideraram o produto melhor que a granola comercial.

\footnotetext{
${ }^{\S}$ Letras diferentes na mesma linha diferem entre si ao nível de $5 \%$ de significância pelo teste de Tukey. $\mathrm{L}^{*}$ representa a claridade $\left(\mathrm{L}^{*}=\right.$
} 0 preto e $\mathrm{L}^{\star}=100$ branco); $\mathrm{a}^{\star}\left(-\mathrm{a}^{\star}\right.$ verde $\mathrm{e}+\mathrm{a}^{\star}$ vermelho) e $\mathrm{b}^{\star}\left(-\mathrm{b}^{\star}\right.$ azul e $+\mathrm{b}^{\star}$ amarelo); $\mathrm{C}^{\star}$ representa a saturação e $\mathrm{h}^{\star}$, a tonalidade. 
Tabela 6 - Valores médios de força aplicada na compressão da granola com bacuri de nove repetições ${ }^{* *} \|$

\begin{tabular}{cr}
\hline Granola com bacuri (repetição) & Força na compressão $(\mathbf{g})^{39}$ \\
\hline A1 & 1979,1 \\
A2 & 1204,4 \\
A3 & 1126,2 \\
A4 & 3024,4 \\
A5 & 3329,9 \\
A6 & 1279,5 \\
A7 & 3251,4 \\
A8 & 3512,9 \\
A9 & 3865,2 \\
\hline
\end{tabular}

\subsection{AVALIAÇÃO SENSORIAL}

O valor médio das notas atribuídas para os atributos aroma, sabor e aceitação global foi 2 (gostei regularmente mais que R). Para cor e textura, os valores foram 1,4 e 1 (gostei ligeiramente mais que R), respectivamente (Figura 4).

A preferência da granola com bacuri pode ser atribuída à maior intensidade da cor amarelo-laranja e à maior crocância, percebidas pelos julgadores quando comparada à granola comercial, o que ratifica os resultados da análise instrumental, mostrando a efetiva correlação. Do exposto, pode-se concluir que o produto com adição de bacuri (polpa e castanha), um fruto não convencional e pouco conhecido no Brasil, foi sensorialmente melhor que a granola comercial.

Figura 4 - Valores médios das notas atribuídas para a granola com adição de bacuri pelo teste sensorial de diferença do controle, em que a granola comercial é o controle (nota 0,0$)$

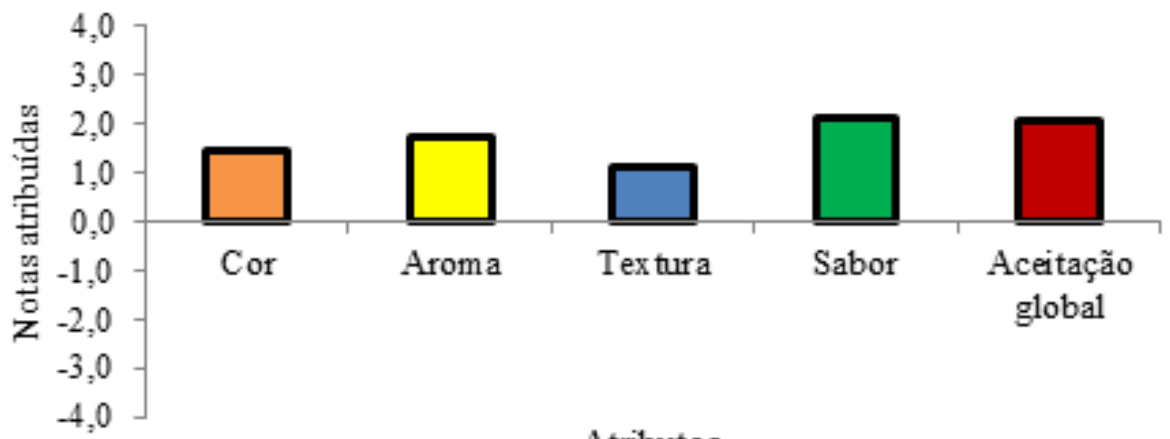

Atributos

$\| \mathrm{A}=$ Amostra de granola com bacuri.

'Valor médio de 200 pontos detectados pela sonda do texturômetro na compressão de cada amostra. 
Na avaliação da intenção de compra da granola com bacuri (Figura 5), observou-se que 56\% dos julgadores expressaram a intenção "certamente compraria”, 22\%, "possivelmente compraria”, 12\%, "talvez comprasse/talvez não comprasse", 6\%, "possivelmente não compraria” e 4\%, "certamente não compraria". Portanto, a frequência dos resultados "certamente compraria" e "possivelmente compraria" mostra que $78 \%$ dos julgadores comprariam ou ao menos pensariam em adquirir o produto desenvolvido caso ele estivesse no mercado.

Diante dos resultados obtidos, pode-se inferir que a adição de polpa e da castanha de bacuri na granola é uma alternativa para o aproveitamento desse fruto ainda pouco aproveitado no Brasil, disponibilizando uma nova opção para o consumidor com atrativos nutritivos, sensoriais e mercadológicos.

Figura 5 - Frequência da intenção de compra para a granola com bacuri

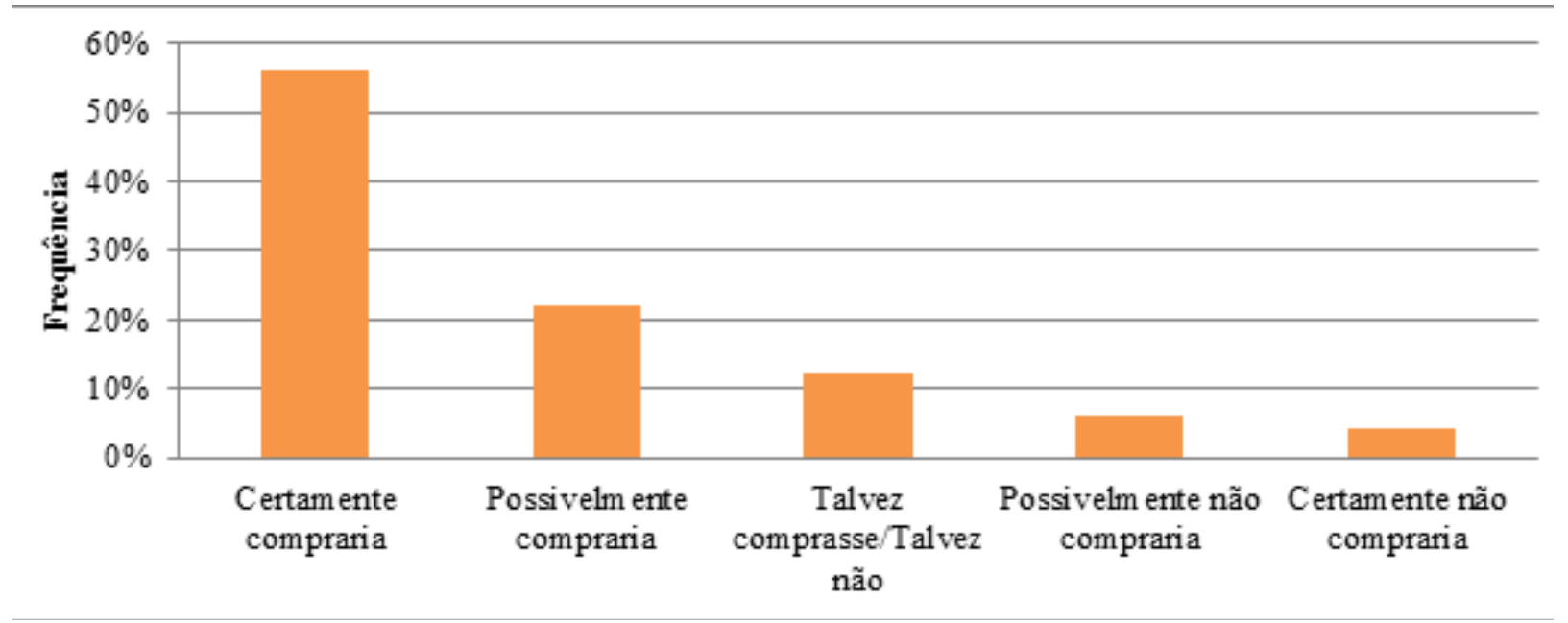

\section{CONCLUSÃO}

Os resultados apresentados no estudo permitem concluir que a polpa e a castanha de bacuri apresentam-se como ingredientes adequados para a formulação de granola, uma vez que demonstraram melhoria nas propriedades de cor, textura, valor nutricional e energético do produto em relação à granola comercial.

A recomendação do consumo de granola com bacuri e iogurte permite aproveitar os benefícios dos constituintes nutricionais presentes no produto e proporciona uma nova opção de alimento rápido que atende às exigências do consumidor que deseja produtos com qualidade sensorial e nutricional associada aos benefícios que eles proporcionam à saúde.

\section{REFERÊNCIAS}

1. Moraes FP, Colla, LM. Alimentos funcionais e nutracêuticos: definições, legislação e benefícios à saúde. Rev Eletrônica Farm. [Internet]. 2006; 2(3): 109-22. 
2. Vidal AM, Dias DO, Martins ESM, Oliveira RS, Nascimento MS, Correia MGS. A ingestão de alimentos funcionais e sua contribuição para a diminuição da incidência de doenças. [Internet]. Cadernos de Graduação 2012; [acesso em 2015 out 20]: 1(15): 43-52. Disponível em: https://periodicos.set.edu.br/index.php/cadernobiologicas/article/view/284/112

3. Bas N, Pathare PB, Catak M, Fitzpatrick JJ, Cronin K, Byrne EP. Mathematical modelling of granola breakage during pipe pneumatic conveying. Powder Technol 2011 Jan; 206(1-2): 170-6.

4. Fujiwara, C. Granola é aliada do intestino e ajuda a controlar o colesterol. [Internet]. 2015 [acesso em 2015 jul 10]. Disponível em: http://www.minhavida.com.br/\%20alimentacao/materias/ 18355-granola-e-aliada-do-intestino-e-ajuda-a-controlar-o-colesterol

5. Martins RC, Filgueiras TS. Arecaceae. In: Cavalcanti TB. Flora do Distrito Federal, Brasil. Brasília, DF: Embrapa Recursos Genéticos e Biotecnologia; 2006; p. 47-82.

6. Lima FF. Microencapsulação do óleo de bacuri por coacervação complexa: Obtenção, caracterização e avaliação biológica. [dissertação]. Dourados: Universidade Federal da grande Dourados; 2014.

7. Sousa MSB, Vieira, LM, Silva MJM, Lima A. Caracterização nutricional e compostos antioxidantes em resíduos de polpas de frutas tropicais. Ciênc Agrotec. 2011; 35(3): 554-9.

8. Gomes FS. Carotenóides: uma possível proteção contra o desenvolvimento de câncer. Rev Nutr. 2007; 20(5): 537-48.

9. Rodriguez-Amaya DB. Quantitative analysis, in vitro assessment of bioavailability and antioxidant activity of food carotenoids - A review. J Food Compos Anal. 2010; 23(7): 726-40.

10. Ribeiro JF, Sano S, Brito MA, Fonseca CEL. Baru (Dipteryx alata Vog.). Jaboticabal: Funep; 2000.

11. Silva AS, Silva DB, Junqueira NTV, Andrade LRM. Frutas nativas dos cerrados. Brasília: Embrapa CPAC; 1994.

12. Ministério da Saúde. Secretaria de Atenção à saúde. Coordenação Geral da Política de Alimentação e Nutrição. Guia alimentar para a população brasileira: promovendo a alimentação saudável. Brasília, DF: Ministério da Saúde; 2006.

13. Ministério da Saúde. Secretaria de Atenção à saúde. Política nacional de alimentação e nutrição. Brasília, DF: Ministério da Saúde; 2003.

14. Silva NDA. Manual de métodos de análise microbiológica de alimentos e água. São Paulo: Varela; 2010. 
15. Brasil. Ministério da Saúde. Secretaria de Vigilância Sanitária. Resolução RDC n. 12, de 02 de janeiro de 2001. Regulamento técnico sobre os padrões microbiológicos para alimentos. [Internet]. [acesso em 2015 jul. 13]. Disponível em: http://portal.anvisa.gov.br/wps/wcm/connect/ a47bab8047458b909541d53fbc4c6735/RDC_12_2001.pdf?MOD=AJPERES

16. Instituto Adolfo Lutz. Normas analíticas do Instituto Adolfo Lutz. São Paulo; 2008.

17. Brasil. Ministério da Saúde. Agência Nacional de Vigilância Sanitária. Resolução-RDC n. 360 de 23 de dezembro de 2003. Dispõe sobre o regulamento técnico de rotulagem nutricional de alimentos embalados. Diário Oficial [da] República Federativa do Brasil, 2003 dez. 17.

18. Anjo DFC. Alimentos funcionais em angiologia e cirurgia vascular. J Vasc Bras. 2004; 3(2): 145 54.

19. Fox PF, McSweeney PLH. Dairy chemistry and biochemistry. London: Blackie; 1998.

20. Souza ML, Menezes HC. Avaliação sensorial de cereais matinais de castanha-do-brasil com mandioca extrusados. Ciênc Tecnol Aliment. 2006; 26(4): 950-5.

21. Magalhães KCP, Alves VC, Peres AP, Sales WB. Análise de coliformes totais e termotolerantes em granola e qualidade higiênico sanitária em casas de cereais de Curitiba - PR. Cad. da Esc. de Saúde [Internet]. 2015 [acesso em 2015 out 14]; 1(13): 105-15. Disponível em: http://revistas. unibrasil.com.br/cadernossaude/index.php/saude/article/view/210

22. Granada G, Rosa V, Zambiasi R, Koetz P. Caracterização de granolas comerciais. Ciênc Tecnol Aliment. 2003; 23(1): 87-91.

23. Santos AAS, Coelho AFS, Carreiro AFS. Avaliação microbiológica de polpas de frutas congeladas. Ciênc Tecnol de Aliment. 2008; 28(4): 913-5.

24. Souza AHP, Gohara AK, Stroher G, Rodrigues AC, Pagamunici LM, Souza NE et al. Estudo multivariado dos macronutrientes em granolas comerciais. In: Anais do $6^{\circ}$ Encontro Nacional de Tecnologia Química, 2013; Maceió. Rio de Janeiro: ABQ; 2013. p. 1. [Internet]. [acesso em 2015 set 3]. Disponível em: http://www.abq.org.br/entequi/2013/trabalhos/50/50-2418-16363. html

25. Park KJ, Yado MKM, Brod FPR. Estudo de secagem de pêra bartlett (Pyrus sp.) em fatias. Ciênc Tecnol Aliment. 2001; 21(3): 288-92.

26. Vitalli A. Importância da atividade de água em alimentos. In: Jardim DCP. I Seminário sobre Atividade de Água em Alimentos. Campinas: ITAL; 1987. 
27. Hall CW. Drying and storage of agricultural crops. Westport: The AVI Publishing Company; 1980.

28. Brooker DB, Bakker-Arkena FW, Hall CW. Drying and storage of grains and oilseeds. New York: AVI; 1992.

29. Enig MG. Lauric oils as antimicrobial agents: Theory of effect, scientific rationale, and dietary applications as adjunct nutritional support for HIV-infected individuals. In: Watson RR, editor. Nutrients and Foods in Aids. 1998. p. 81-97.

30. DeLany JP, Windhauser MM, Champagne CM, Bray GA. Differential oxidation of individual dietary fatty acids in humans. [Internet]. Am J Clin Nutr. 2000 [acesso em 2015 set 3]; 72(4): 905-11. Disponível em: http://ajcn.nutrition.org/content/72/4/905.long

31. St-Onge MP, Jones PJH. Greater rise in fat oxidation with medium-chain triglyceride consumption relative to long-chain triglyceride is associated with lower initial body weight and greater loss of subcutaneous adipose tissue. Int J Obes. 2003; 27(12): 1565-71. doi: 10.1038/ sj.ijo.0802467

32. Vögler O, Lopez-Bellan A, Alemany R, Tofé S, González M, Quevedo J, Escriba PV. Structureeffect relation of $\mathrm{C} 18$ long-chain fatty acids in the reduction of body weight in rats. Int J Obes. 2007; 32(3): 464-73. doi: 10.1038/sj.ijo.0803768

33. Coppini LZ, Waitzberg DL, Campos FG, Harb-Gama A. Fibras Alimentares e Ácidos Graxos de Cadeia Curta. In: Waitzberg, D.L. Nutrição Oral, Enteral e Parenteral na Prática Clínica. São Paulo: Atheneu; 2004. p. 79-94.

34. Brasil. Ministério da Saúde. Agência Nacional de Vigilância Sanitária - Anvisa. Resolução RDC N. 359, de 23 de dezembro de 2003. Regulamento Técnico de Porções de Alimentos Embalados para Fins de Rotulagem Nutricional. 2003 dez.

35. Maclaren DS, Frigg M. Sigght and life manual on vitamin A deficiency disorders Task Force Sight and Life. Basel: Switzerland; 2001.

36. Olson JA. Bioavailability of carotenoids. Archivos latinoamericanos de Nutrición 1999; 49(1): 21-5.

37. Krinsky NI. The biological properties of carotenoids. Pure Appl Chem 1994; 66: 1003-10.

38. Mayne ST. Beta carotene, carotenoids, and disease prevention in humans. FASEB J. 1996; 10: 690-701. 
39. Brasil. Ministério da Saúde, Agência Nacional de Vigilância Sanitária - Anvisa. Resolução RDC n. 269, de 22 de setembro de 2005. O regulamento técnico sobre a ingestão diária recomendada (idr) de proteína, vitaminas e minerais. [Internet]. [acesso em 2015 set 29]. Disponível em: http://portal.anvisa.gov.br/wps/wcm/connect/1884970047457811857dd53fbc4c6735/ RDC_269_2005.pdf?MOD=AJPERES

40. Tabela Brasileira de Composição de Alimentos. Campinas: NEPA - Unicamp; 2011. 161 p. [Internet]. [acesso em 2015 set 5]. Disponível em: http://www.unicamp.br/nepa/taco/contar/ taco_4_edicao_ampliada_e_revisada

41. Brasil. Ministério da Agricultura, Pecuária e Abastecimento. Secretaria de Defesa Agropecuária. Padrões de Identidade e Qualidade de Leites Fermentados. Instrução Normativa n. 46, de 23 de outubro de 2007.

42. Vasconcelos MAS, Melo Filho AB. Conservação de Alimentos. [Internet]. [acesso em 2015 set 29]. Recife: EDUFRPE; 2010. Disponível em: http://redeetec.mec.gov.br/images/stories/pdf/ eixo_prod_alim/tec_alim/181012_con_alim.pdf

43. Teixeira ACP, Mourthé K, Alexandre DP, Souza MR, Penna CFAM. Qualidade do Iogurte Comercializado em Belo Horizonte. Leite \& Derivados 2000; 1(51): 32-9.

44. Ferreira CLLF, Malta HL, Dias AS, Guimarães A, Jacob FE, Cunha RM, Careli RT, Pereira S, Ferreira SER. Verificação da qualidade físico-química e microbiológica de alguns iogurtes vendidos na região de Viçosa. Rev Inst Latic Cândido Tostes 2001; 56(321): 152-8.

45. Associação Portuguesa dos Nutricionistas. O iogurte - para saber mais. Porto: APN; 2013 maio [coleção E-books APN]. [Internet]. [acesso em 2015 nov 7]. Disponível em: http://www.apn.org. pt/documentos/ebooks/Ebook_Iogurte.pdf

46. Brasil. Ministério da Saúde. Agência Nacional de Vigilância Sanitária - Anvisa. Resolução RDC n. 360, de 23 de dezembro de 2003. Regulamento Técnico sobre Rotulagem Nutricional de Alimentos Embalados, tornando obrigatória a rotulagem nutricional. [Internet]. [acesso em 2015 set 29]. Disponível em: http://portal.anvisa.gov.br/wps/wcm/connect/ec3966804ac02cf1962abfa337abae9d/Resolucao_RDC_n_360de_23_de_dezembro_de_2003.pdf?MOD=AJPERES

47. Moritz AR. Existe cor em nossas vidas - a colorimetria aplicada em nossos dias. São Paulo: Mundo da cor; 2011.

Data da submissão: 24 de maio de 2016 Avaliado em: 05 de dezembro de 2016 (AVALIADOR A) Avaliado em: 07 de dezembro de 2016 (AVALIADOR B)

Aceito em: 12 de dezembro de 2016 